Agricultural Engineering, Research Papers, 2014, Vol. 46, No 1, 4-13 p.

\title{
APPLICATION OF SOFT COMPUTING TECHNIQUES TO MOTION CONTROL OF UNDERWATER ROBOTIC VEHICLE
}

\author{
Jerzy Garus, Józef Małecki \\ Polish Naval Academy, Faculty of Mechanical and Electrical Engineering \\ e-mail: j.garus@amw.gdynia.pl,j.malecki@amw.gdynia.pl
}

Received 2014-06-12, accepted 2014-09-10

\begin{abstract}
Using of artificial intelligence techniques to steering of underwater robotic vehicle along a reference path is considered in the paper. For the tracking, the way-point line of sight scheme is applied. Command signals are generated by an autopilot consisting of four independent controllers with a fuzzy control law implemented. Parameters of the controllers are tuned by means of genetic algorithms. A quality of control is tested without and in presence of environmental disturbances. Selected results of computer simulations are inserted to demonstrate quality of the approach.
\end{abstract}

\section{Introduction}

Underwater Robotics has known an increasing interest in the last years. Main benefits of usage of Underwater Robotic Vehicles (URVs) can be removing men from the dangers of the underwater environment and reduction in cost of exploration of deep seas. Contemporary, it is common to use URVs to accomplish such missions as inspection of coastal and off-shore structures, cable maintenance, as well as hydrographical and biological surveys. In the military field they are employed in such tasks as surveillance, intelligence gathering, torpedo recovery and mine counter measures.

The URV is considered being a floating platform carrying tools required for performing various functions. The most often used are manipulator arms with interchangeable end-effectors, cameras, scanners, sonars, etc. Automatic control of such object is a difficult problem caused by its nonlinear dynamics. Moreover, the dynamics can change according to the alteration of configuration to be suited to the mission. In order to cope with these difficulties the control system should be flexible. Interesting reviews of classical and modern techniques adopted to control the dynamic behaviour of unmanned underwater vehicles have been provided in (Craven et al., 1998; Fossen, 1994, 2002; Song et al., 2003).

Nowadays, fuzzy systems find wide practical applications, ranging from soft regulatory control in consumer products to accurate control and modeling of complex nonlinear systems (Driankow et al., 1996; Garus, 2005; Kacprzyk, 1997; Ross, 2005; Yager, Filev, 1994). In this paper we design the fuzzy autopilot to track-keeping control of the underwater vehicle tuning its parameters by means of genetic algorithms. 


\section{Equations of Motion}

The following vectors describe the motion of marine vehicles of six degrees of freedom (DOFs) (Fossen, 1994):

$$
\begin{aligned}
\boldsymbol{\eta} & =[x, y, z, \Phi, \Theta, \Psi]^{T} \\
\mathbf{v} & =[u, v, w, p, q, r]^{T} \\
\boldsymbol{\tau} & =[X, Y, Z, K, M, N]^{T}
\end{aligned}
$$

where:

$\boldsymbol{\eta} \quad-$ vector of position and orientation in the inertial frame;

$x, y, z$ - coordinates of position;

$\Phi, \Theta, \Psi$ - coordinates of orientation (Euler angles);

$\mathbf{v} \quad-$ the linear and angular velocity vector with coordinates in the body fixed frame;

$u, v, w$ - linear velocities along longitudinal, transversal and normal axes;

$p, q, r$ - angular velocities about longitudinal, transversal and normal axes;

$\tau-$ vector of forces and moments acting on the robot in the body-fixed frame;

$X, Y, Z$ - forces along longitudinal, transversal and normal axes;

$K, M, N$ - moments about longitudinal, transversal and normal axes.

The nonlinear dynamic equations of motion can be expressed as (Fossen, 2002):

$$
\begin{aligned}
& \mathbf{M} \dot{\mathbf{v}}+\mathbf{C}(\mathbf{v}) \mathbf{v}+\mathbf{D v}+\mathbf{g}(\boldsymbol{\eta})=\boldsymbol{\tau} \\
& \dot{\boldsymbol{\eta}}=\mathbf{J}(\boldsymbol{\eta}) \mathbf{v}
\end{aligned}
$$

where:

M - inertia matrix (including added mass);

$\mathbf{C}(\mathbf{v})$ - matrix of Coriolis and centripetal terms (including added mass);

$\mathbf{D}(\mathbf{v})$ - hydrodynamic damping and lift matrix;

$\mathbf{g}(\boldsymbol{\eta})$ - vector of gravitational forces and moments;

$\mathbf{J}(\eta)$ - velocity transformation matrix between vehicle and earth fixed frames.

\section{Tracking and Coordinate Systems}

The conventional URV moves in horizontal plane with some variation due to diving. Hence, it can operate in crab-wise manner in four DOFs with small roll and pitch angles that can be neglected during normal operations. Therefore, it is purposeful to regard spatial motion of the vehicle as superposition of two displacements: movement in the horizontal plane and movement in the vertical plane. It allows us to decrease the number of controlled movements from six to four.

It is convenient to define three coordinate systems when analysing the tracking system for the vehicle's motion in the horizontal plane (see Fig. 1) (Fossen, 1994, Garus, Kitowski, 2004): 
1. the global coordinate system $\mathrm{O}_{0} \mathrm{X}_{0} \mathrm{Y}_{0}$ (called also the earth-fixed frame);

2. the local coordinate system OXY (fixed to the body of the vehicle);

3. the reference coordinate system $P_{i} X_{i} Y_{i}$ (system is not fixed).

A main goal of the autopilot steering motion in the horizontal plane is to minimize mean square deviations $\Delta y$ and $\Delta \Psi$ measured in the system $\mathrm{P}_{i} \mathrm{X}_{i} \mathrm{Y}_{i}$, where (see Fig. 1):

- $\Delta y$ is a perpendicular distance of the robot's centre of gravity to the predefined path;

- $\Delta \Psi$ is a local heading angle defined as the angle between the track reference line and the robot's centreline.

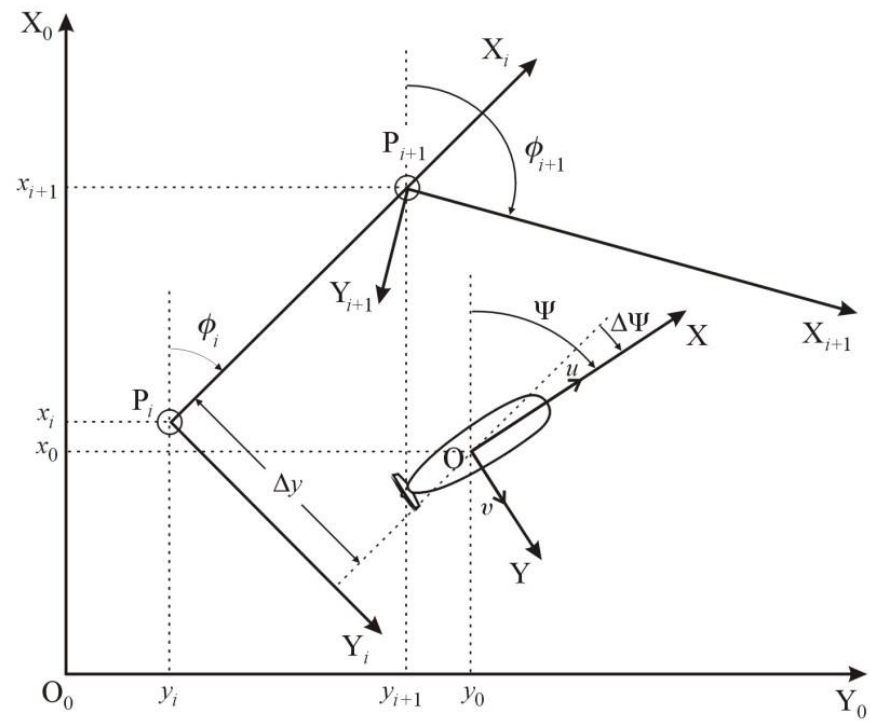

Fig. 1. Coordinate systems applied to description of robot's horizontal motion: $\mathrm{O}_{0} \mathrm{X}_{0} \mathrm{Y}_{0}-$ global system, $\mathrm{OXY}$ - local system, $\mathrm{P}_{\mathrm{i}} \mathrm{X}_{\mathrm{i}} \mathrm{Y}_{\mathrm{i}}$ - reference system

A form of the assumed cost function describes the following expression:

$$
J_{H}=\min \sum_{t}\left(\lambda_{y} \Delta y^{2}(t)+\lambda_{\Psi} \Delta \Psi^{2}(t)\right)
$$

where:

$$
\Delta y(t)=-\sin \left(\phi_{i}\right)\left(x_{0}(t)-x_{i}\right)+\cos \left(\phi_{i}\right)\left(y_{0}(t)-y_{i}\right),
$$

$x_{0}(t), y_{0}(t)-\quad$ current coordinates of the robot's centre of gravity in the global system $\mathrm{O}_{0} \mathrm{X}_{0} \mathrm{Y}_{0}$,

$x_{i}, y_{i} \quad-\quad$ coordinates of the point $\mathrm{P}_{i}$ in the system $\mathrm{O}_{0} \mathrm{X}_{0} \mathrm{Y}_{0}$,

$\phi_{i} \quad-\quad$ angle of rotation of the reference coordinate system with respect to the global one: 


$$
\phi_{i}=\arctan \left[\frac{y_{i+1}-y_{i}}{x_{i+1}-x_{i}}\right]
$$

$\Delta \Psi(t)=\Psi(t)-\phi_{i}$,

$\Psi(t) \quad-\quad$ instantaneous course of the robot in the system $\mathrm{O}_{0} \mathrm{X}_{0} \mathrm{Y}_{0}$,

$t \quad-\quad$ time,

$\lambda_{y}, \lambda_{\Psi}-$ constant coefficients.

A movement in the vertical plane (see Fig. 2), i.e. a linear displacement along $z$-axis from the point $\mathrm{P}_{i}$ with a coordinate $z_{i}$ to the point $\mathrm{P}_{i+1}$ with a coordinate $z_{i+1}$, regarded in the earth-coordinate system $\mathrm{O}_{0} \mathrm{X}_{0} \mathrm{Z}_{0}$ (note that the $\mathrm{Z}$ axis of the local coordinate system is parallel to $Z_{0}$ axis). A main task of the autopilot steering the motion in this plane is to minimize mean square deviations of the vehicle's center of gravity from a demanded draught $z_{d}$ :

$$
J_{V}=\min \sum_{t} \Delta z^{2}(t)
$$

where:

$$
\Delta z(t)=z_{0}(t)-z_{d}
$$

$z_{0}(t)$ - a current coordinate of the robot's centre of gravity in the system $\mathrm{O}_{0} \mathrm{X}_{0} \mathrm{Z}_{0}$

$$
z_{d}=z_{i+1} \text {. }
$$

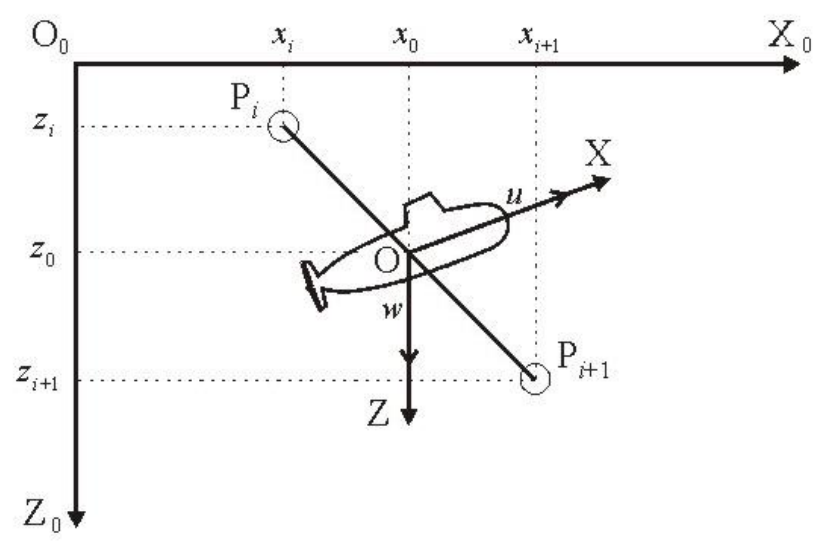

Fig. 2. Coordinate systems used in vertical motion: $\mathrm{O}_{0} \mathrm{X}_{0} \mathrm{Z}_{0}$ - earth-fixed system, $\mathrm{OXZ}-$ body-fixed system

Each time the vehicle location $\left(x_{0}(t), y_{0}(t), z_{0}(t)\right)$ at the time $t$ satisfies:

$$
\left[x_{i}-x_{0}(t)\right]^{2}+\left[y_{i}-y_{0}(t)\right]^{2}+\left[z_{i}-z_{0}(t)\right]^{2} \leq \rho^{2}
$$


where $\rho$ is a circle of acceptance, the next way-point should be selected, the reference coordinate system $\mathrm{P}_{i} \mathrm{X}_{i} \mathrm{Y}_{i}$ changed into $\mathrm{P}_{i+1} \mathrm{X}_{i+1} \mathrm{Y}_{i+1}$, an angle of rotation is $\phi_{i+1}$ calculated and the vehicle's position updated corresponding to the new reference coordinate system.

\section{Fuzzy Control System}

The control system was designed under the following assumptions:

1. the vehicle can move with varying linear velocities $u, v, w$ and the angular velocity $r$;

2. coordinates of position $x, y, z$ and the heading $\Psi$ are measurable;

3. the desired path is a broken line defined by set of way-points $\mathrm{P}_{1}, \mathrm{P}_{2}, \mathrm{P}_{3}$, etc. with coordinates respectively $\left(x_{1}, y_{1}, z_{1}\right),\left(x_{2}, y_{2}, z_{2}\right),\left(x_{3}, y_{3}, z_{3}\right)$, etc.;

4. the command signal consists of only four components $\tau=[X, Y, Z, N]$.

A structure of such control system is depicted in Fig. 3.

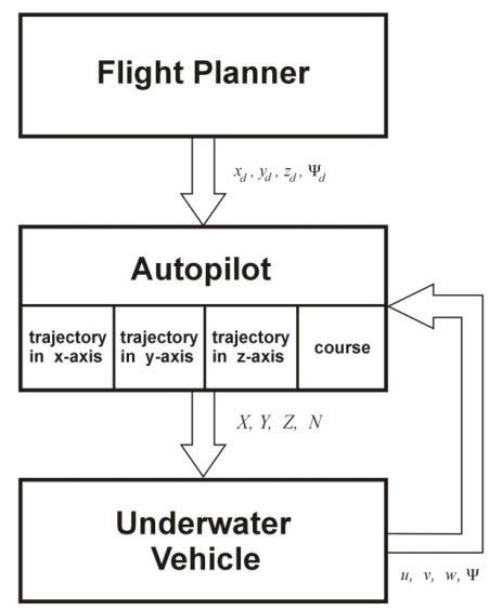

Fig. 3. A general structure of the control system

The regarded autopilot consists of four independent fuzzy proportional derivative (FPD) controller, adopted from ((Driankow et al., 1996), working in configuration presented in Fig. 4.

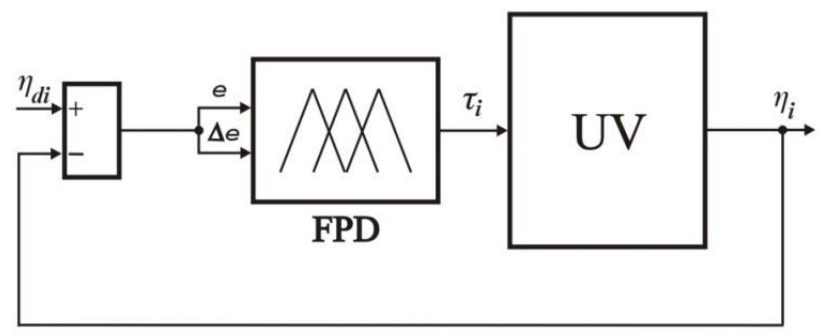

Fig.4. A fuzzy control structure 
Membership functions of fuzzy sets of input variables, i.e. the error signal $e(t)=\eta_{d}-\eta(t)$ and the derived change in error $\Delta e(t)=\eta(t)-\eta(t-1)$, as well as the command signal $\tau(t)$ are shown in Fig. 5, respectively. There is used the following notation: $\mathrm{N}$ - negative, $\mathrm{Z}$ - zero, $\mathrm{P}$ - positive, $\mathrm{S}$ - small, $\mathrm{M}$ - medium and $\mathrm{B}-$ big.
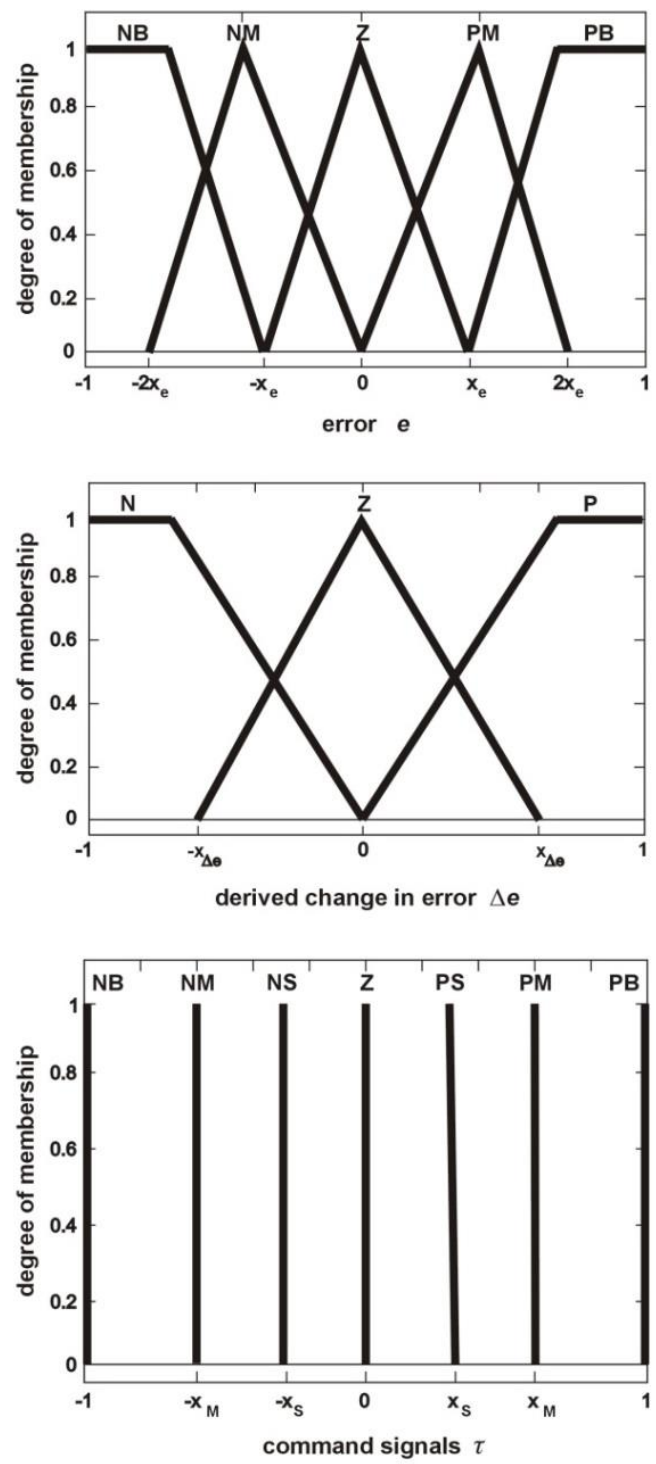

Fig. 5. Membership functions for fuzzy sets: error signal $e$, derived change in error $\Delta e$ and command signal $\tau$ 
Presented in Table 1 rules from the Mac Vicar-Whelan's standard base of rules have been chosen as the control ones (Yager, Filev, 1994). Unknown parameters of the membership functions were determined using genetic algorithms (GA), which basis on the Darwin's principle of reproduction and survival of the fittest (Goldberg, 1989; Michalewicz, 1994). In general, this technique manipulates sets of individuals (solutions) by using genetic operators (selection, reproduction, crossover and mutation) in order to propose better ones. Chromosomes represent the individuals in a population. The chromosome consists of four values that corresponded to unknown parameters of the membership functions (see Fig. 6). Their tuning ranges were defined as follows: $0<x_{e} \leq 0.5,0.5<x_{\Delta e}<1$, $0<x_{M} \leq 0.5,0.25<x_{S} \leq 1$.

\begin{tabular}{|l|l|l|l|}
\hline$x_{e}$ & $x_{\Delta e}$ & $x_{S}$ & $x_{M}$ \\
\hline
\end{tabular}

Fig. 6. Structure of the chromosome

Table 1. The fuzzy controller's base of rules

\begin{tabular}{|c|c|c|c|c|c|c|}
\cline { 3 - 7 } \multicolumn{2}{c|}{} & \multicolumn{6}{c|}{ Error signal $e$} \\
\cline { 3 - 7 } \multicolumn{2}{c|}{} & $\mathrm{NB}$ & $\mathrm{NM}$ & $\mathrm{Z}$ & $\mathrm{PM}$ & $\mathrm{PB}$ \\
\hline \multirow{2}{*}{$\begin{array}{c}\text { Derived change } \\
\text { in error } \Delta e\end{array}$} & $\mathrm{~N}$ & $\mathrm{NB}$ & $\mathrm{NM}$ & $\mathrm{NS}$ & $\mathrm{Z}$ & $\mathrm{PS}$ \\
\cline { 2 - 7 } & $\mathrm{Z}$ & $\mathrm{NM}$ & $\mathrm{NS}$ & $\mathrm{Z}$ & $\mathrm{PS}$ & $\mathrm{PM}$ \\
\cline { 2 - 7 } & $\mathrm{B}$ & $\mathrm{NS}$ & $\mathrm{Z}$ & $\mathrm{PS}$ & $\mathrm{PM}$ & $\mathrm{PB}$ \\
\hline
\end{tabular}

The parameters of the membership functions of the fuzzy controllers, calculated for the following configuration of the GA: population -20 , crossover 0.8 , mutation -0.05 and generation -100 , shows Table 2 . 
Table 2. Parameters of membership functions of controllers

\begin{tabular}{|c|c|c|c|c|}
\cline { 2 - 5 } \multicolumn{1}{c|}{} & \multicolumn{4}{c|}{ Controller } \\
\cline { 2 - 5 } & $\begin{array}{c}\text { motion } \\
\text { along } x \text {-axis }\end{array}$ & $\begin{array}{c}\text { motion } \\
\text { along } y \text {-axis }\end{array}$ & $\begin{array}{c}\text { motion } \\
\text { along } z \text {-axis }\end{array}$ & course \\
\hline$x_{e}$ & 0.14 & 0.19 & 0.11 & 0.39 \\
\hline$x_{\Delta e}$ & 0.87 & 0.63 & 0.75 & 0.52 \\
\hline$x_{S}$ & 0.25 & 0.40 & 0.46 & 0.38 \\
\hline$x_{M}$ & 0.89 & 0.74 & 0.83 & 0.65 \\
\hline
\end{tabular}

\section{Simulation Study}

Simulation experiments were conducted to validate the performance of the developed fuzzy autopilot using the MATLAB/Simulink environment. An applied model of the URV bases on the real construction of the underwater vehicle called UKWIAL, designed and built for the Polish Navy.

The URV has an open-frame structure and is: $1.5 \mathrm{~m}$ long, $1.0 \mathrm{~m}$ wide, and $0.8 \mathrm{~m}$ high. It has a dry weight of $140 \mathrm{~kg}$ and a maximum operating depth of $300 \mathrm{~m}$. The vehicle is equipped with four thrusters responsible for surge, sway and yaw motion in the horizontal plane, and two vertical thrusters for heave motion. Pitch and roll motion are left uncontrolled, since the metacentric height is sufficient large to provide static stability. It means that the object is controllable in 4 DOF.

Simulations were done for the following assumptions:

1. the way-point line of sight scheme is incorporated for the tracking of desired path;

2. the vehicle has to follow the desired trajectory beginning from $(10 \mathrm{~m}, 10 \mathrm{~m}, 0 \mathrm{~m})$, passing target way-points: $(10 \mathrm{~m}, 10 \mathrm{~m},-5 \mathrm{~m}),(10 \mathrm{~m}, 90 \mathrm{~m},-$ $5 \mathrm{~m}),(30 \mathrm{~m}, 90 \mathrm{~m},-5 \mathrm{~m}),(30 \mathrm{~m}, 10 \mathrm{~m},-5 \mathrm{~m}),(60 \mathrm{~m}, 10 \mathrm{~m}, 5 \mathrm{~m}),(60 \mathrm{~m}, 90 \mathrm{~m},-5 \mathrm{~m})$, $(60 \mathrm{~m}, 90 \mathrm{~m}, 15 \mathrm{~m}), \quad(60 \mathrm{~m}, \quad 10 \mathrm{~m},-15 \mathrm{~m}), \quad(30 \mathrm{~m}, 10 \mathrm{~m}, \quad-15 \mathrm{~m}), \quad(30 \mathrm{~m}, 90 \mathrm{~m},-$ $15 \mathrm{~m}),(10 \mathrm{~m}, 90 \mathrm{~m},-15 \mathrm{~m})$, and ending in $(10 \mathrm{~m}, 10 \mathrm{~m},-15 \mathrm{~m})$;

3. the turning point is reached when the vehicle is inside the $2.0 \mathrm{~m}$ circle of acceptance;

4. a sea current interact on the vehicle with average speed $0.3 \mathrm{~m} / \mathrm{s}$ and direction $135^{\circ}$.

Some results of numerical experiments are shown in Fig. 7. They show that the proposed autopilot with the fuzzy control law enhanced good tracking control of the desired path in the spatial motion. 

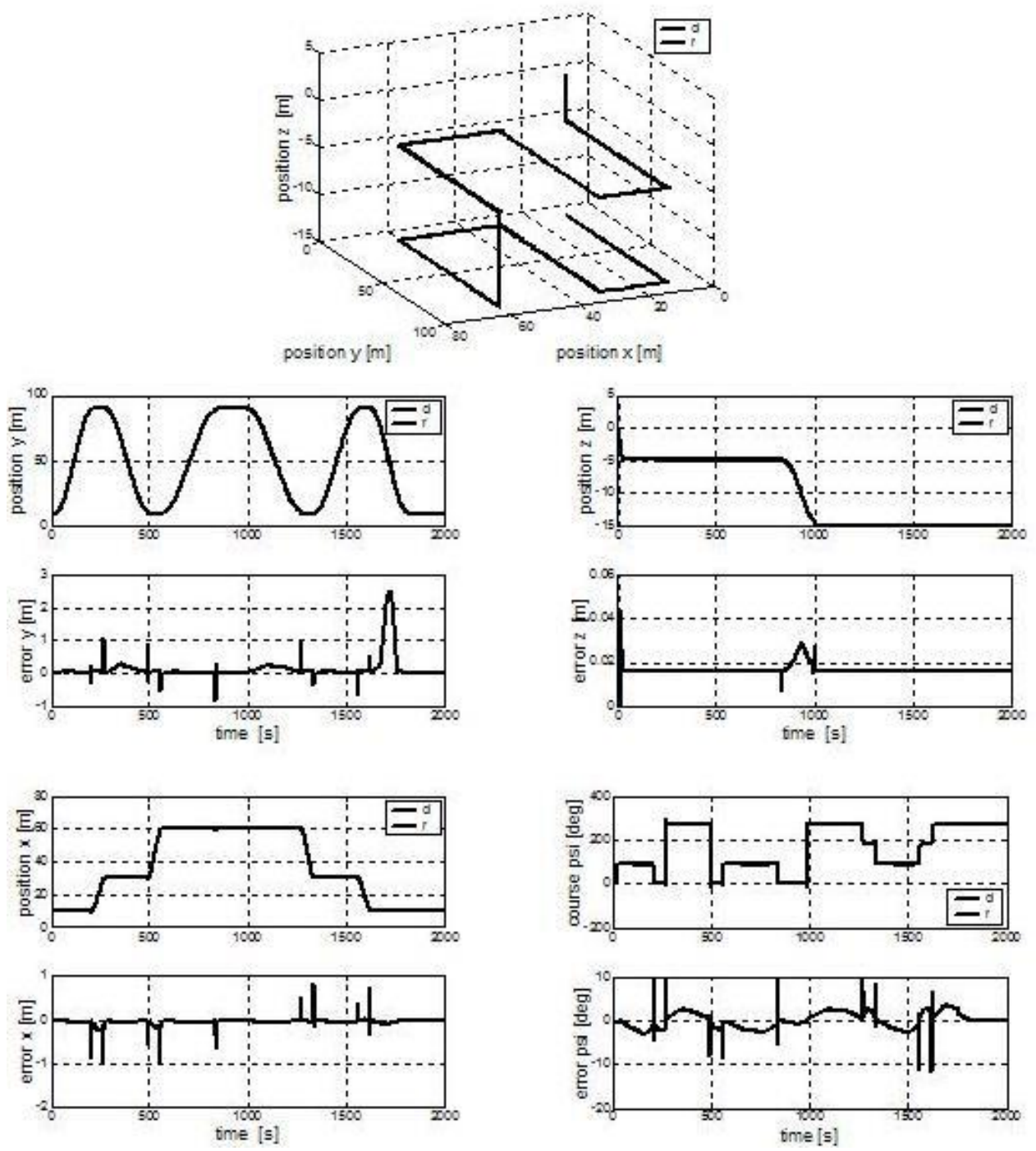

Fig. 7. Simulation of 3D motion under interaction of environmental disturbances: desired (d) and real (r) paths (upper plot), x-, y-, z-position and error of position (1st $\div 2$ nd left plots), course and error of course (1st right plot), commands (2nd right plot) 


\section{Conclusions}

In the paper the fuzzy control system for the underwater vehicle was presented. From the obtained results, it can be concluded that the proposed approach provides the autopilot being robust and having good performance.

An additional advantage is usefulness of the described algorithm to practical applications due its numerical simplicity.

Further works are needed to identify the best fuzzy structure of the autopilot and test the robustness of this approach in a real world.

\section{Acknowledgement}

This work was partially supported under a research grant (2012-2015) by the Polish National Centre for Research and Development.

\section{References}

1. Craven, J.; Sutton, R.; Burns, R. 1998. Control Strategies for Unmanned Underwater Vehicles, Journal of Navigation, 51(2), 79-105.

2. Driankov, D.; Hellendoorn, H.; Reinfrank, M. 1996. An Introduction to Fuzzy Control. Berlin: Springer-Verlag.

3. Fossen, T.I. 1994. Guidance and Control of Ocean Vehicles. Chichester: Wiley and Sons.

4. Fossen, T.I. 2002. Marine Control Systems. Trondheim: Marine Cybernetics AS.

5. Garus, J.; Kitowski, Z. 2004. Tracking Autopilot for Underwater Robotic Vehicle. In: Cagnol, J.; Zolesio, J.P. (Eds.). Information Processing: Recent Mathematical Advances in Optimization and Control. Paris: Presses de l'Ecole des Mines de Paris.127-138.

6. Garus, J. 2005. Fuzzy Trajectory Control Design for Underwater Robot. In: $4^{\text {th }}$ Conference of the European Society for Fuzzy Logic and Technology Proceedings, 7-9 September 2005, Barcelona, 232-239.

7. Goldberg, D. 1989. Genetic Algorithms in Search, Optimisation and Machine Learning. New York: Adison-Wesley.

8. Kacprzyk, J. 1997. Multistage Fuzzy Control. John Wiley and Sons.

9. Michalewicz, Z. 1994. Genetic Algorithms + Data Structures = Evolution Programs. New York: Springer-Verlag.

10.Ross, T. 2005. Fuzzy Logic with Engineering Applications. New York: Wiley and Sons.

11.Yager, R.R.; Filev, D. P. 1994. Essential of Fuzzy Modelling and Control. New York: Wiley and Sons.

12.Song, F.; An, P.E.; Folleco, A. 2003. Modelling and Simulation of Autonomous Underwater Vehicles: Design and Implementation. IEEE Journal of Oceanic Engineering, 28, 283-296. 